\section{Efeitos de diferentes estratégias de treinamento combinado na forca muscular e na potência aeróbia dé idosos: uma revisão sistemática}

\author{
Effects of different concurrent training \\ strategies in muscular strength and aerobic \\ power in the elderly: a systematic review
}

Roger Freier Nascimento

Ana Carolina Kanitz ${ }^{1}$

Luiz Fernando Martins Kruel ${ }^{1}$

\section{RESUMO}

O objetivo do estudo foi revisar os efeitos de diferentes estratégias metodológicas de treinamento combinado na força e na potência aeróbia de indivíduos idosos saudáveis. A busca incluiu as bases de dados da MEDLINE (via Pubmed) e COCHRANE. A partir dessa busca, 3035 artigos tiveram seus títulos/resumos avaliados, destes 3026 foram excluídos e nove foram selecionados para análise. Foram incluídos dois estudos a partir das referências de outros estudos, totalizando 11 estudos para a leitura na íntegra. Após a leitura dos estudos, foram excluídos quatro por não estarem de acordo com os critérios de elegibilidade, restando sete para análise. Foram observadas melhoras significativas na força com o treinamento combinado. Entretanto, a realização simultânea de um treinamento aeróbio em cicloergômetro e em intensidades próximas ao segundo limiar ventilatório $\left(\mathrm{FC}_{\mathrm{LV} 2}\right)$ e de força (nesta ordem de execução) pode apresentar um efeito de interferência nas respostas de força de membros inferiores. $\mathrm{O}$ desempenho da potência aeróbia não foi afetado quando o treino de força foi realizado antes. Além disso, uma frequência semanal de duas vezes apresenta iguais benefícios em comparação a uma frequência maior. Desta forma, parece que o treinamento aeróbio em cicloergômetro em intensidades baseadas no $\mathrm{FC}_{\mathrm{LV} 2}$ realizado antes do treinamento de força, provoca interferência nos ganhos de força muscular de membros inferiores e o treinamento de força antes do aeróbio não afeta o desempenho da potência aeróbia. Ainda, uma frequência semanal de treinamento combinado menor apresenta benefícios em amplitudes semelhantes a uma frequência maior.

\section{PALAVRAS-CHAVE}

Envelhecimento; Treinamento de força; Capacidade física.

\begin{abstract}
The aim of the study was to review the effects of different methodological strategies of combined training in strength and aerobic power performance of healthy elderly subjects. A literature search was conducted using MEDLINE (via PubMed) and COCHRANE. A total of 3035 manuscripts had their titles/abstracts evaluated, these 3026 were excluded and nine were selected for analysis. Have been included two studies from references to other studies, totaling 11 studies for reading in full. After reading the studies, four were excluded because they are not in accordance with the eligibility criteria, leaving nine studies for analysis Significant improvements were observed in muscle strength with the combined training. However, when the simultaneous attainment of an aerobic training on a cycle ergometer and intensities close to the point corresponding to the second ventilatory threshold (VT2) and force (in this order of execution) may have an interference effect on muscle strength responses of the lower limbs. The performance of aerobic power was not affected when strength training was accomplished before. In addition, a frequency twice weekly bas the same benefits as compared to a bigher frequency. Thus, it seems that aerobic training on a cycle ergometer using as intensity of VT2 done before strength training causes interference in muscle strength of the lower limbs gains. Perform strength training before the aerobic training did not shave the performance of aerobic power. Further; a weekly frequency lower combined training has similar benefits to a bigher frequency ranges.
\end{abstract}

Rev Bras Ativ Fís Saúde p. 329-339 DOI

http://dx.doi.org/10.12820/rbafs.v.20n4p329

1 Escola de Educação Física - Universidade Federal do Rio Grande do Sul

\title{
KEYWORDS
}

Aging; Strength Training; Physical Ability. 


\section{INTRODUÇÃOO}

O processo de envelhecimento humano é associado com declínios em funções fisiológicas importantes para a capacidade funcional dos indivíduos. Dentre essas mudanças, podemos citar a redução da potência aeróbia, diminuição da massa muscular e consequente perda de força e resistência muscular ${ }^{1,2}$, alterações que refletem diretamente na dependência funcional dos indivíduos. Porém, o grau dessas alterações no organismo com o envelhecimento depende do quanto ativo é o indivíduo. Quanto mais ativo for, mais lentamente ocorrerão esses declínios ${ }^{3}$.

Com o envelhecimento há uma redução significativa na capacidade aeróbia, o que reflete em uma menor capacidade do corpo em transportar oxigênio para os tecidos ${ }^{4}$. Segundo dados da literatura, o consumo máximo de oxigênio $\left(\mathrm{VO}_{2 \operatorname{máx}}\right)$ reduz em torno de $1 \%$ ao ano entre os 25 e os 75 anos de idade ${ }^{5}$. Esse decréscimo no $\mathrm{VO}_{2 \max }$ parece estar relacionado com as alterações hormonais recorrentes do envelhecimento e o declínio da frequência cardíaca máxima ${ }^{4}$. Entretanto, segundo Matsudo, Matsudo e $\mathrm{Neto}^{4}$ o declínio no $\mathrm{VO}_{2 \text { máx }}$ parece estar mais relacionado ao nível habitual de atividade física do que as alterações fisiologias decorrentes da idade. Neste sentido, os mesmos autores destacam que é requerido um mínimo de $15 \mathrm{ml} \cdot \mathrm{kg}^{-1} \cdot \mathrm{min}^{-1}$ de potência aeróbia para manter as demandas fisiológicas necessárias para viver de forma independente e funcional ${ }^{4}$.

Além disso, com o avanço da idade há significativas alterações fisiológicas no sistema muscular, sendo este mais aparente a partir dos 50 anos de idade e mais acentuado em indivíduos sedentários ${ }^{4}$. Com o envelhecimento, as fibras musculares atrofiam e morrem, enquanto outras são substituídas por tecido adiposo e conjuntivo, ocorrendo um aumento desse tecido e do colágeno intersticial na musculatura do idoso ${ }^{6}$. Esse processo reflete em uma diminuição da massa muscular (sarcopenia) e consequentemente reduz a força, a resistência muscular e a velocidade de contração ${ }^{6}$. Todos esses declínios deixam os indivíduos mais propensos a adquirir doenças crônico-degenerativas, problemas articulares, maiores riscos de fraturas com consequente incapacidade e dependência funcional ${ }^{4}$.

Neste sentindo, três estratégias de treinamento têm sido utilizadas para minimizar esses declínios fisiológicos em idosos: 1) o treinamento aeróbio com o objetivo de melhorar a capacidade cardiorrespiratória, cardiovascular, aumentar o gasto calórico e reduzir os estoques de gordura corporal; 2) o treinamento de força com o objetivo de melhorar a força muscular e preservar ou aumentar a massa magra ${ }^{7}$;) e a combinação desses treinamentos, denominado treinamento combinado, com o intuito de se alcançar ambos os benefícios. Porém, estudos apontam que a execução de treinamento aeróbio antes do treinamento de força, numa mesma sessão, pode gerar conflitos nas adaptações neurais decorrentes do treinamento de força ${ }^{8-11}$, processo conhecido como efeito de interferência ${ }^{8}$. Por outro lado, alguns estudos na literatura não encontram esse efeito e defendem a ideia da realização de exercícios aeróbios e de força em uma mesma sessão para promover os benefícios proporcionados por ambos os treinamentos ${ }^{12-15}$. As diferentes estratégias metodológicas utilizadas entre os estudos podem ter influenciado nas divergências dos resultados encontrados, principalmente em relação a ordem de execução dos exercícios, número de sessões semanais, intensidade dos treinamentos e a escolha dos exercícios. 
Assim, uma vez que os exercícios de força e aeróbios são muito procurados por idosos devidos aos inúmeros benefícios já mencionados, torna-se importante revisar as metodologias utilizadas nestes estudos para auxiliar os profissionais de educação física na prescrição de treinamento para essa população. Desta forma, o objetivo do presente estudo foi verificar, através de uma revisão sistemática, quais os efeitos de diferentes estratégias metodológicas de treinamento combinado no desempenho da força muscular e potência aeróbia em indivíduos idosos saudáveis acima de 60 anos.

A hipótese do estudo é que o treinamento combinado apresenta benefícios importantes na força e na potência aeróbica de idosos e que estes são semelhantes aos do treinamento de força e aeróbico realizado de forma isolada. No entanto, acredita-se que essas melhoras serão dependentes das diferentes estratégias de treinamento utilizada.

\section{MÉTODOS}

\section{Critérios de elegibilidade}

Foram incluídos artigos originais a partir do ano de 2010 que avaliassem idosos (idade $>60$ anos) saudáveis que fossem submetidos a um treinamento combinado de força e potência aeróbia. Ao serem selecionados artigos publicados nesse período, o objetivo é verificar o estado da arte mais atual sobre a temática em questão. Os desfechos incluídos foram todos aqueles relacionados às respostas na força muscular e potência aeróbia. Foram excluídos os estudos que apresentavam falta de dados ou dados incompletos, em língua que não o inglês ou português, que não apresentasse um grupo controle ou comparador e estudos crossover sem os dados da primeira fase.

\section{Estratégia de busca}

A busca dos estudos foi realizada nos seguintes bancos de dados: MEDLINE (acessado via PubMed) e COCHRANE. Em adição, foi realizada a busca manual a partir das referências dos estudos encontrados por meio das bases de dados. A estratégia de busca completa utilizada para o PubMed pode ser observada na Tabela 1 .

TABELA 1 - Estratégia de busca utilizada no PubMed.

\begin{tabular}{|c|c|}
\hline$\# 1$ & "aged" [mesh] OR "aged" OR "elderly" OR “older people" OR "older adults" \\
\hline \#2 & $\begin{array}{l}\text { "resistance training" [mesh] OR "training, resistance" OR "Strength Training" OR "Training, } \\
\text { Strength" OR "Weight-Lifting Strengthening Program" OR "Strengthening Program, Weight- } \\
\text { Lifting" OR "Strengthening Programs, Weight-Lifting" OR "Weight Lifting Strengthening } \\
\text { Program" OR "Weight-Lifting Strengthening Programs" OR "Weight-Lifting Exercise } \\
\text { Program" OR "Exercise Program, Weight-Lifting" OR "Exercise Programs, Weight-Lifting" } \\
\text { OR "Weight Lifting Exercise Program" OR "Weight-Lifting Exercise Programs" OR "Weight- } \\
\text { Bearing Strengthening Program" OR "Strengthening Program, Weight-Bearing" OR } \\
\text { "Strengthening Programs, Weight-Bearing" OR "Weight Bearing Strengthening Program" } \\
\text { OR "Weight-Bearing Strengthening Programs" OR "Weight-Bearing Exercise Program" OR } \\
\text { "Exercise Program, Weight-Bearing" OR "Exercise Programs, Weight-Bearing" OR "Weight } \\
\text { Bearing Exercise Program" OR "Weight-Bearing Exercise Programs" }\end{array}$ \\
\hline \#3 & $2010 / 01 / 01$ to present \\
\hline \#4 & \#1 AND \#2 AND \#3 \\
\hline
\end{tabular}

\section{Seleção dos estudos e extração dos dados}

Para a seleção dos estudos, os títulos e os resumos de todos os estudos encon- 
trados pela estratégia de busca foram avaliados. Aqueles que não forneceram informações suficientes para os critérios de inclusão e exclusão foram selecionados para a leitura e avaliação do texto na íntegra. Nesta segunda etapa foi feita a leitura dos estudos completos e selecionados aqueles que estavam de acordo com os critérios de elegibilidade. As avaliações foram realizadas por dois revisores de forma independente e as discordâncias entre os revisores foram solucionadas por consenso.

\section{Avaliação do risco de viés}

A qualidade metodológica de cada estudo foi avaliada, individualmente, por dois investigadores. Os seguintes itens foram considerados na análise dos estudos incluídos: geração de sequência de randomização, sigilo de alocação, cegamento, cegamento dos avaliadores dos desfechos, análise por intenção de tratar e descrição das perdas e exclusões. Além disso, a qualidade metodológica dos estudos foi avaliada com base na escala PEDro ${ }^{16}$, baseada na lista de Delphi ${ }^{17}$. Essa escala possui 11 questões, no entanto dez são pontuadas. Cada critério é pontuado de acordo com a sua presença ou ausência no estudo em questão, sendo contado um ponto para cada critério presente (exceto o primeiro). Todos os estudos incluídos foram avaliados de forma independente por dois revisores e quando em discordância chegava-se a um consenso.

\section{Cálculo da diferença entre as médias e do tamanho do efeito}

A diferença entre as médias e o tamanho do efeito foi calculado para a variável consumo de oxigênio de pico representando a potência aeróbia e para a variável de uma repetição máxima de extensão de joelho representando a força muscular. Os cálculos foram realizados no software Excel, a diferença entre as médias foi calculada pela subtração da média do valor pós pela média do valor pré-treinamento e o tamanho do efeito foi calculado pela divisão da diferença entre as médias e o desvio padrão agrupado.

\section{RESULTADOS}

\section{Descrição dos estudos}

A busca inicial resultou em 3035 estudos nas bases de dados, destes 3026 foram excluídos a partir da leitura do título e resumo por não se enquadrarem nos critérios de elegibilidade, restando nove estudos para análise. Foram incluídos dois estudos a partir das referências de outros estudos, totalizando 11 estudos para a leitura na íntegra. Após a leitura dos estudos, foram excluídos quatro por não terem avaliados apenas idosos, restando sete artigos que terão seus resultados descritos nesta revisão (Figura 1).

\section{Diferença entre as médias e o tamanho do efeito}

Os valores das diferenças entre as médias podem ser visualizados na Tabela 1. Para o consumo de oxigênio de pico as maiores diferenças entre as médias foram encontrados nos estudos de Cadore et al. ${ }^{8,11}$ para o grupo combinado (pré: $27 \pm 3.7 \mathrm{ml} \cdot \mathrm{kg}^{-1} \cdot \mathrm{min}^{-1}$; pós: $32.9 \pm 2.7 \mathrm{ml} \cdot \mathrm{kg}^{-1} \cdot \mathrm{min}^{-1}$, diferença entre as médias: $5,9 \mathrm{ml} \cdot \mathrm{kg}^{-1} \cdot \mathrm{min}^{-1}$ ) e os menores valores foram encontrados no estudo de Fisher et al. ${ }^{19}$ para o grupo combinado na ordem aeróbio-força realizado uma vez por semana (pré: $21.6 \pm 5 \mathrm{ml} \cdot \mathrm{kg}^{-1} \cdot \mathrm{min}^{-1}$; pós: $22 \pm 5 \mathrm{ml} \cdot \mathrm{kg}^{-1} \cdot \mathrm{min}^{-1}$, diferença 
TABELA 1 - Diferença entre as médias para as variáveis consumo de oxigênio de pico (VO2pico) e uma repetição máxima de extensão de joelho (1RMext) nos grupos GFA (grupo aeróbio-força), GAF (grupo força-aeróbio), GC (grupo combinado), GF (grupo força), GA (grupo aeróbio), 2x GFA (grupo força-aeróbio duas vezes por semana), 3x GFA (grupo força-aeróbio três vezes por semana), 1x GAF (grupo aeróbio-força uma vez por semanas), 2x GAF (grupo aeróbio-força duas vezes por semanas) e 3x GAF (grupo aeróbio-força três vezes por semanas).

\begin{tabular}{lcccccccccc}
\hline & GFA & GAF & GC & GF & GA & $\begin{array}{c}2 X \\
\text { GFA }\end{array}$ & $\begin{array}{c}3 X \\
\text { GFA }\end{array}$ & $\begin{array}{c}1 X \\
\text { GAF }\end{array}$ & $\begin{array}{c}2 X \\
\text { GAF }\end{array}$ & $\begin{array}{c}3 X \\
\text { GAF }\end{array}$ \\
\hline $\mathrm{VO}_{2 \text { pico }}\left(\mathrm{ml}^{\prime} \mathrm{kg}^{-1} \cdot \mathrm{min}^{-1}\right)$ & 2,1 & 2,2 & 5,9 & 1,0 & 5,0 & 4,9 & 3,6 & 0,4 & 1,6 & 0,5 \\
\hline $1 \mathrm{RM}_{\text {ext }}(\mathrm{kg})$ & 23,4 & 15,6 & 26,5 & 39,4 & 13,4 & 20,0 & 21,0 & 6,0 & 8,0 & 6,0 \\
\hline
\end{tabular}

3035 estudos identificados

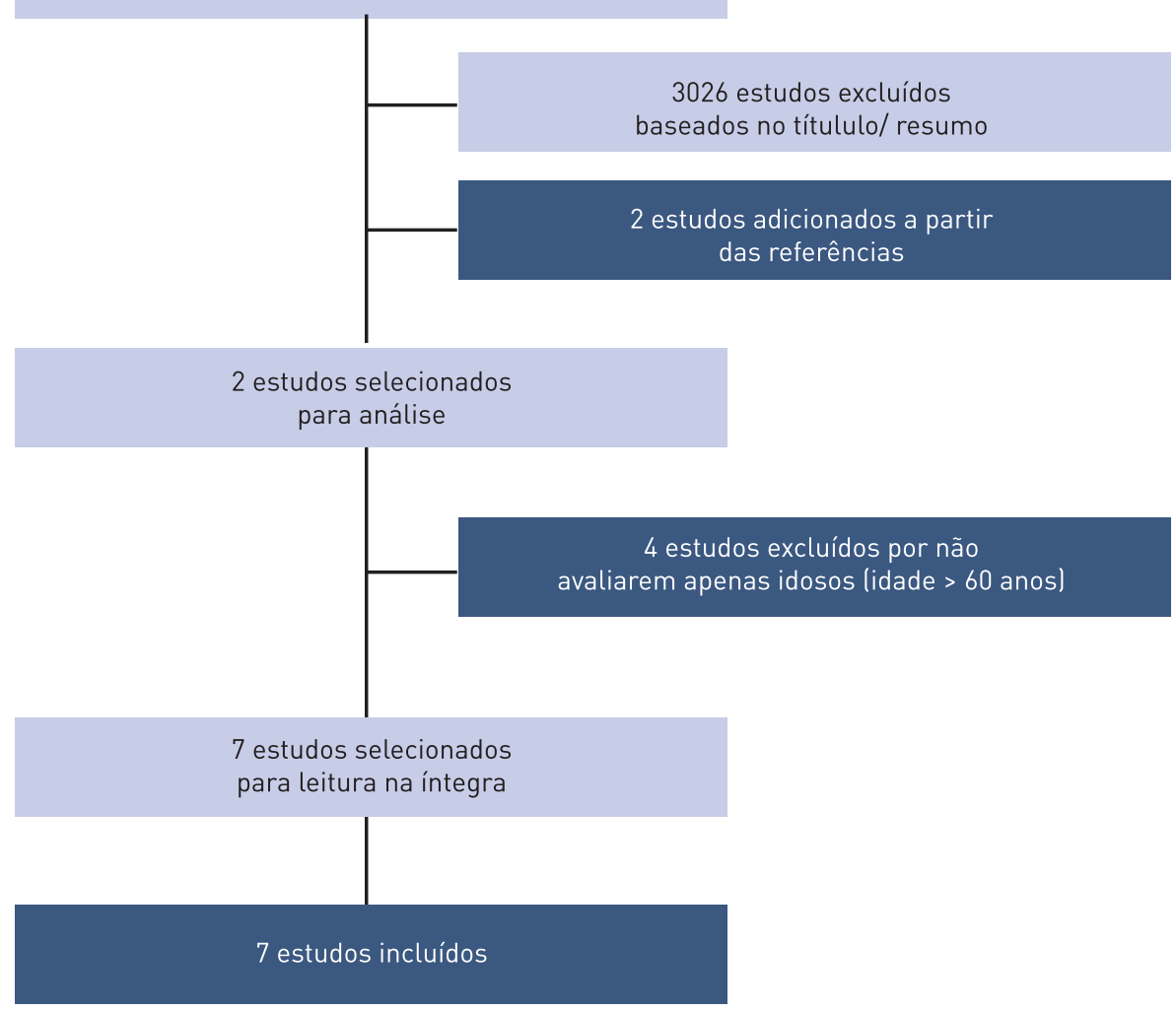

FIGURA 1 - Fluxograma dos estudos incluídos na revisão.

entre as médias: $\left.0,4 \mathrm{ml} \cdot \mathrm{kg}^{-1} \cdot \mathrm{min}^{-1}\right)$. Para a variável de uma repetição máxima de extensão de joelho o grupo que apresentou os maiores valores foi o grupo força, também do estudo de Cadore et al. ${ }^{11}$ (pré: $58.3 \pm 10 \mathrm{~kg}$; pós: $97.7 \pm 10 \mathrm{~kg}$, diferença entre as médias: $39,4 \mathrm{~kg}$ ) e os menores valores foram encontrados no estudo de Fisher et al. ${ }^{19}$ nos grupos que realizaram o treinamento combinado uma (pré: $31 \pm 9 \mathrm{~kg}$; pós: $37 \pm 10 \mathrm{~kg}$, diferença entre as médias: $6 \mathrm{~kg}$ ) e três vezes por semana (pré: $33 \pm 6 \mathrm{~kg}$, pós: $39 \pm 6 \mathrm{~kg}$, diferença entre as médias: $6 \mathrm{~kg}$ ).

O tamanho do efeito foi calculado para as variáveis consumo de oxigênio de pico e uma repetição máxima de extensão de joelho, representando a potência aeróbia e a força muscular, respectivamente. $\mathrm{O}$ tamanho do efeito auxilia a entender melhor a importância do resultado obtido no estudo.

Os resultados mostram que quanto maior o tamanho do efeito maior é o impacto da intervenção na variável analisada. A Figura 2 apresenta o tamanho do efeito para o consumo de oxigênio de pico para todos os grupos que avalia- 
ram essa variável nos estudos incluídos. Podemos observar um alto tamanho de efeito para o grupo combinado ${ }^{8,11}$, seguido do grupo combinado (ordem força-aeróbio) com frequência semanal de duas vezes ${ }^{18}$ e grupo aeróbio ${ }^{8,11}$. O grupo que apresentou o menor tamanho de efeito foi o combinado na ordem aeróbio-força realizado uma vez por semana.

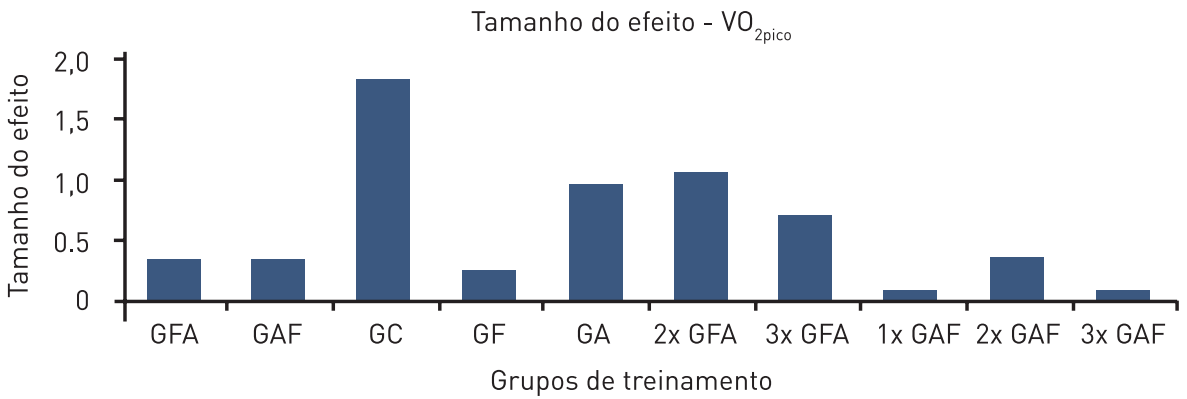

FIGURA 2 - Tamanho do efeito para a variável consumo de oxigênio de pico $\left(\mathrm{VO}_{2 \text { pico }}\right)$ nos grupos GFA (grupo aeróbio-força), GAF (grupo força-aeróbio), GC (grupo combinado), GF (grupo força), GA (grupo aeróbio), 2x GFA (grupo força-aeróbio duas vezes por semana), 3x GFA (grupo força-aeróbio três vezes por semana), 1x GAF (grupo aeróbio-força uma vez por semanas), 2x GAF (grupo aeróbio-força duas vezes por semanas) e 3x GAF (grupo aeróbio-força três vezes por semanas).

Além disso, a Figura 3 apresenta o tamanho do efeito para a variável uma repetição máxima de extensão de joelho para todos os grupos que avaliaram essa variável nos estudos incluídos. Neste caso, o grupo força ${ }^{11}$ apresentou o maior tamanho de efeito, seguido do grupo combinado ${ }^{9-11}$. O grupo que apresentou menor tamanho de efeito foi novamente o combinado na ordem aeróbio-força realizado uma vez por semana.

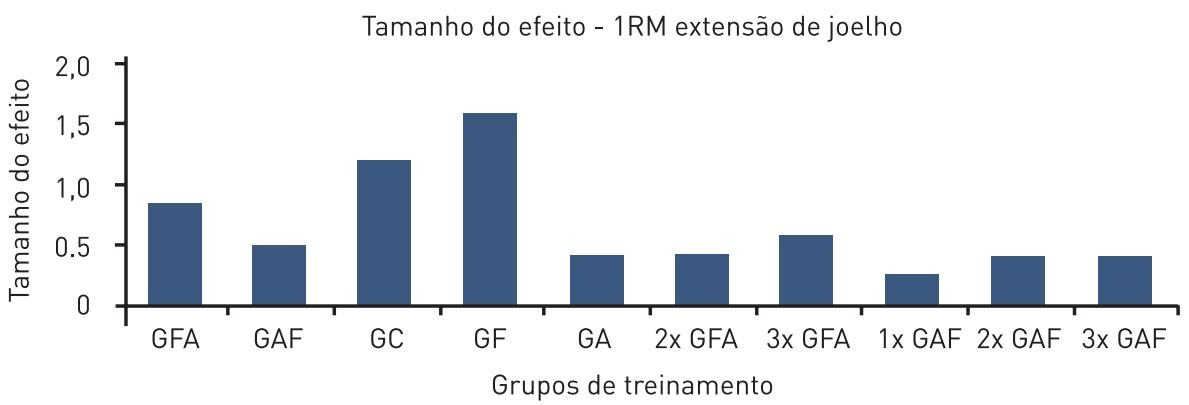

FIGURA 3 - Tamanho do efeito para a variável uma repetição máxima (1RM) de extensão de joelho nos grupos GFA (grupo aeróbio-força), GAF (grupo força-aeróbio), GC (grupo combinado), GF (grupo força), GA (grupo aeróbio), 2x GFA (grupo força-aeróbio duas vezes por semana), 3x GFA (grupo força -aeróbio três vezes por semana), 1x GAF (grupo aeróbio-força uma vez por semanas), 2x GAF (grupo aeróbio-força duas vezes por semanas) e 3x GAF (grupo aeróbio-força três vezes por semanas).

\section{Efeito das intervenções}

Os estudos incluídos tiveram um tempo mínimo de intervenção de 10 semanas e máximo de 16 semanas. Cinco estudos utilizaram um período de treinamento de 12 semanas, com frequência de treino de três vezes por semana, em dias não consecutivos e treino de força e aeróbio na mesma sessão ${ }^{8-11,14}$. O estudo que realizou o treinamento em 10 semanas teve frequência semanal de treino de duas e três vezes, em dias não consecutivos e treino de força e aeróbio na mesma sessão ${ }^{18}$. O estudo que teve o tempo de intervenção de 16 semanas, o treinamento de força e aeróbico foi realizado em dias diferentes e com frequência semanal de treino de uma, duas e três vezes ${ }^{19}$. 
Em relação à amostra, seis estudos tiveram um número semelhante de participantes (22-26 participantes) ${ }^{8-11,18}$ e um estudo teve uma quantia significativamente acima dos demais (63 participantes) ${ }^{19}$. Na soma das amostras de todos os estudos avaliados tivemos um total de 207 participantes. A média de idade dos participantes foi de 65 anos. A amostra de cinco estudos ${ }^{8-11,18}$ foi composta somente de homens, e de dois estudos ${ }^{14,19}$ de mulheres. Nenhum estudo avaliou as duas populações de forma conjunta.

Em relação às metodologias utilizadas para o treinamento aeróbio, cinco estudos ${ }^{8-11,18}$ utilizaram somente cicloergômetro no treinamento aeróbio, com intensidade que variou de $80-100 \%$ da frequência cardíaca do segundo limiar ventilatório $\left(\mathrm{FC}_{\mathrm{LV} 2}\right)$ e tempo de duração de 20 a 30 minutos. Em um estudo $^{14}$, os participantes realizaram treinamento aeróbio somente em esteira com intensidade que variou de 65 a $85 \%$ da frequência cardíaca máxima $\left(\mathrm{FC}_{\text {máx }}\right)$ e tempo de duração de 20 a 30 minutos. Em uma das pesquisas ${ }^{19}$ os participantes poderiam optar em realizar o treinamento aeróbio na esteira ou cicloergômetro com intensidade que variou de $65-85 \%$ da $\mathrm{FC}_{\text {máx }}$ e tempo de duração de 20 a 40 minutos.

Em relação ao treinamento de força, os quatro estudos de Cadore et al. ${ }^{8-}$ ${ }^{11}$ e o de Campos et al. ${ }^{14}$ utilizaram metodologias semelhantes com aumento gradual do volume e da intensidade do treinamento ao longo da periodização, utilizando 2 a 3 séries com carga que variaram de 18-20 Repetições Máximas (RM), progredindo para 15-17RM, 12-14RM, 8-10RM e 6-8RM com execução até a falha. De forma semelhante, o treinamento de força realizado no estudo de Ferrari et al. ${ }^{18}$ consistiu na execução também de 2 a 3 séries com repetições realizadas até a falha (repetições máximas) que progrediram ao longo da periodização de 12-10 RM a 10-8 RM e a 8-6 RM. Por fim, o estudo de Fisher et al. ${ }^{19}$ teve um volume de treino de força menor, com 1 a 2 séries de 8-10 RM, mas com foco na potência muscular. Todos os estudos realizaram exercícios para os principais grupos musculares de membros superiores e inferiores no treinamento de força.

Dos sete estudos, cinco tiveram como objetivo avaliar a ocorrência ou não do efeito de interferência no treinamento combinado ${ }^{8-11,14}$. Destes, três avaliaram o efeito da ordem da sessão, $0^{9,14}$ e dois compararam o treinamento combinado com o treinamento de força e aeróbio realizado de forma isola$\mathrm{da}^{8,11}$. Além disso, dois estudos ${ }^{16,19}$ avaliaram os efeitos do volume semanal de treinamento combinado. Ferrari et al. ${ }^{18}$ compararam grupos que realizaram o treinamento combinado em uma mesma sessão de duas e três vezes por semanas e Fisher et al. ${ }^{19}$ comparou grupos de frequências de uma, duas e três vezes por semana, realizando os treinamentos de força e aeróbio em sessões diferentes. A Tabela 2 mostra as características dos estudos incluídos.

\section{Risco de viés}

Dos sete estudos incluídos, sete (100\%) descreveram a realização de geração de sequência aleatória, nenhum estudo relatou alocação sigilosa e cegamento. $\mathrm{O}$ cegamento dos avaliadores dos desfechos foi relatado em dois estudos (28,5\%). Apenas dois estudos $(28,5 \%)$ descreveram perdas e exclusões e nenhum estudo forneceu informações sobre a realização de intenção de tratar (Tabela 3). Desta forma, apesar dos resultados positivos encontrados nos estudos avaliados, estes devem ser interpretados com cautela devido ao alto risco de viés metodológico. Além disso, 
com base na avaliação da qualidade da evidência através da escala PEDro, dos sete estudos selecionados a maior pontuação foi atribuída aos estudos de Cadore et al. ${ }^{9}$ e Cadore et al. ${ }^{10}$ (7/10). Em relação aos demais estudos, três pontuaram 6/10 (Cadore et al..$^{8,11}$ e Ferrari et al..$^{18}$ ) e dois 5/10 (Fisher et al. ${ }^{19}$; Campos et al. ${ }^{14}$ ). Enfatizamos que os principais itens que não foram pontuados estão relacionados ao cegamento da distribuição da amostra, do cegamento do grupo em que os sujeitos estavam inseridos, cegamento do professor e dos avaliadores. Neste contexto, sugere-se uma atenção maior dos estudos referente ao cegamento do processo de randomização e dos avaliadores, entretanto, o cegamento dos alunos e dos professores torna-se inviável tratando-se de intervenções com exercício físico.

TABELA 2 - Adaptações neuromusculares e cardiorrespiratórias ao treinamento combinado.

\begin{tabular}{|c|c|c|c|c|}
\hline ESTUDO & AMOSTRA & PERÍODO e FREQUÊNCIA & VOLUME e INTENSIDADE & RESULTADOS \\
\hline Cadore et al. ${ }^{9}$ & $\begin{array}{l}26 \text { homens idosos } \\
\text { saudáveis }(64,7 \pm \\
4,1 \text { anos). }\end{array}$ & 12 sem; GFA x GAF: 3x/sem. & $\begin{array}{l}\text { TF: } 2 \text { a } 3 \text { séries; } 18-20 R M \\
\text { a 6-8RM. } \\
\text { TA: } 30 \text { min em } \\
\text { cicloergômetro, } 80 \text { a } 100 \% \\
\text { da FC } \text { Lv2. }\end{array}$ & $\begin{array}{l}\uparrow \mathrm{VO}_{2 \text { pico }} \mathrm{GFA}=\mathrm{GAF} \\
\uparrow \mathrm{QM} \text { dos extensores de joelho } \\
\mathrm{GFA}>\mathrm{GAF} .\end{array}$ \\
\hline Cadore et al. ${ }^{10}$ & $\begin{array}{l}26 \text { homens idosos } \\
\text { saudáveis }(64,7 \pm \\
4,1 \text { anos). }\end{array}$ & 12 sem; GFA x GAF: 3x/sem. & $\begin{array}{l}\text { TF: } 2 \text { a } 3 \text { séries; } 18-20 \mathrm{RM} \\
\text { a 6-8RM. } \\
\text { TA: } 30 \text { min em } \\
\text { cicloergômetro, } 80 \text { a } 100 \% \\
\text { da FC } \text { Lv2. }\end{array}$ & 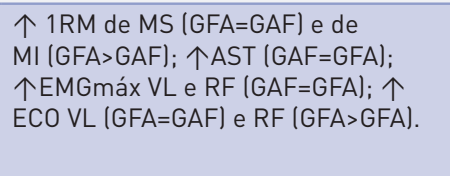 \\
\hline Cadore et al. ${ }^{11}$ & $\begin{array}{l}23 \text { homens idosos } \\
\text { saudáveis }(65 \pm 4 \\
\text { anos). }\end{array}$ & $\begin{array}{l}12 \text { sem; } 3 x / \text { sem; GC lordem } \\
\text { aeróbico-força) x GF x GA. }\end{array}$ & $\begin{array}{l}\text { TF: } 2 \text { a } 3 \text { séries; } 18-20 \mathrm{RM} \\
\text { a 6-8RM; } \\
\text { TA: } 20-30 \text { min em } \\
\text { cicloergômetro, } 80 \text { a } 100 \% \\
\text { FC }_{\text {LV2 }}\end{array}$ & 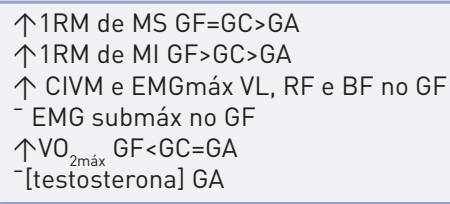 \\
\hline Cadore et al. ${ }^{8}$ & $\begin{array}{l}23 \text { homens idosos } \\
\text { saudáveis }(65 \pm 4 \\
\text { anos). }\end{array}$ & $\begin{array}{l}12 \text { sem; } 3 x / \text { sem GC lordem } \\
\text { aeróbico-força) x GF x GA. }\end{array}$ & $\begin{array}{l}\text { TF: } 2 \text { a } 3 \text { séries; } 18-20 R M \\
\text { a 6-8RM. } \\
\text { TA: } 30 \text { min em } \\
\text { cicloergômetro, } 80 \text { a } 100 \% \\
\text { da FC } \text { Lv2. } \text {. }\end{array}$ & $\begin{array}{l}\uparrow W_{\text {méx }} \text { e } \uparrow V_{2 \text { pico }} \text { em GC e GA; } \\
\text { diminuição atividade eletromiografica } \\
\text { do RF em } 50 \text { W em GA, } 75 \text { e } 10 \text { W em } \\
\text { GA e GC e no VL para os três grupos } \\
\text { em } 100 W .\end{array}$ \\
\hline Campos et al. ${ }^{14}$ & $\begin{array}{l}22 \text { mulheres idosas } \\
\text { fisicamente ativas } \\
\text { (>60 anos). }\end{array}$ & $\begin{array}{l}12 \text { sem, 3x/sem; GA x GF x } \\
\text { GFA x GAF }\end{array}$ & $\begin{array}{l}\text { TF: } 2 \text { a } 3 \text { séries; } 18-20 \text { a } \\
\text { 4-6RM. } \\
\text { TA: } 20 \text { a } 30 \text { minutos em } \\
\text { esteira, } 65 \text { a } 85 \% \text { da } \mathrm{FC}_{\text {máx }} \text {. }\end{array}$ & $\begin{array}{l}\text { = tempo no teste de } 6 \text { min, no 1RM } \\
\text { supino e força de pressão manual, } \\
\text { sem diferença entre os grupos; } \\
\uparrow 1 \mathrm{RM} \text { de flexão de joelho. }\end{array}$ \\
\hline Ferrari et al. ${ }^{18}$ & $\begin{array}{l}24 \text { homens } \\
\text { idosos saudáveis } \\
\text { previamente } \\
\text { treinados ( } 65+4 \\
\text { anos). }\end{array}$ & $\begin{array}{l}10 \text { sem; GFA }: 2 x / \text { sem; }_{\text {GFA }} \text { : } \\
\text { 3x/sem. TF e TA na mesma } \\
\text { sessão. }\end{array}$ & $\begin{array}{l}\text { TF: } 2 \text { a } 3 \text { séries; } 12-10 R M \text { a } \\
\text { 10-8RM a 8-6RM. } \\
\text { TA: } 30 \text { min em } \\
\text { cicloergômetro, } 85 \text { e } 95 \% \\
\text { da FC } \text { LV2. }^{\text {. }}\end{array}$ & 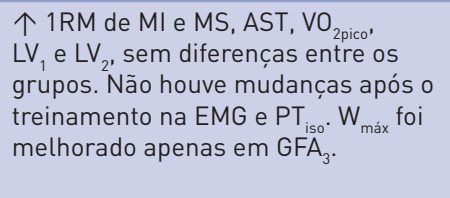 \\
\hline Fisher et al. ${ }^{19}$ & $\begin{array}{l}63 \text { mulheres idosas } \\
\text { saudáveis (60-77 } \\
\text { anos). }\end{array}$ & $\begin{array}{l}16 \text { sem; G1A1F (1x sem TA + } \\
\text { 1x sem TF) x G2A2F (2x sem } \\
\text { TA + } 2 x \text { sem TF) x G3A3F ( } 3 x \\
\text { sem TA + 3x sem TF) }\end{array}$ & $\begin{array}{l}\text { TF: } 1 \text { a } 2 \text { séries de } 8-10 R M / \\
\text { potência. } \\
\text { TA: } 20 \text { a } 40 \text { min em } \\
\text { cicloergômetro ou esteira, } \\
65-80 \% \text { FC }_{\text {máx }}\end{array}$ & $\begin{array}{l}\text { 个1RM leg press, extensão e flexão } \\
\text { de joelho, supino e ombros, sem } \\
\text { diferença entre os grupos. } \\
\text { 个VO }{ }_{2 \text { pico, }} \text { sem diferença entre os } \\
\text { grupos. }\end{array}$ \\
\hline
\end{tabular}

个: aumento; ${ }^{-}$: redução; min: minutos; 1RM: 1 repetição máxima; sem: semana; x/sem: número de sessões por semana; GF: grupo treinamento de força; TF: treinamento de força; GA: grupo treinamento aeróbio; TA: treinamento aeróbio; GAF: grupo de treinamento aeróbio e força; GFA: grupo de treinamento força e aeróbio; GC: grupo de treinamento combinado; QM: qualidade muscular; MS: membros superiores; MI: membros inferiores; EMG: sinal eletromiográfico; $\mathrm{PT}_{\text {iso }}$ : pico de torque isométrico; $A S T$ : aérea de secção transversal; $F_{\mathrm{LV}_{1}}$ e $\mathrm{FC}_{\mathrm{LV} 2}$ : frequência cardíaca correspondente ao primeiro e segundo limiar ventilatório: respectivamente; VL: vasto lateral; RF: reto femoral; BF: bíceps femoral; $L_{1}$ e $L_{2}$ : primeiro e segundo limiar ventilatório: respectivamente; ECO: economia; $\mathrm{VO}_{2 \max }$ : consumo máximo de oxigênio; $\mathrm{VO}_{2 \text { pico }}$ : consumo de pico de oxigênio; $\mathrm{W}_{\text {máx }}$ : carga aeróbica máxima; CIVM: contração isométrica voluntária máxima. 
TABELA 3 - Avaliação do risco de viés.

\begin{tabular}{|c|c|c|c|c|c|c|}
\hline Estudo, ano & $\begin{array}{l}\text { Geração da } \\
\text { sequência aleatória }\end{array}$ & Alocação sigilosa & Cegamento & $\begin{array}{l}\text { Cegamento } \\
\text { avaliadores dos } \\
\text { desfechos }\end{array}$ & $\begin{array}{l}\text { Descrição de } \\
\text { perdas e exclusões }\end{array}$ & $\begin{array}{l}\text { Análise por } \\
\text { Intenção de tratar }\end{array}$ \\
\hline Cadore et al. 2010 & Sim & Não informado & Não informado & Não informado & Não informado & Não informado \\
\hline Cadore et al. 2011 & Sim & Não informado & Não informado & Não informado & $\operatorname{sim}$ & Não informado \\
\hline Cadore et al. 2012 & Sim & Não informado & Não informado & Sim & Não informado & Não informado \\
\hline Cadore et al. 2012 & Sim & Não informado & Não informado & $\operatorname{Sim}$ & Não informado & Não informado \\
\hline Ferrari et al. 2013 & Sim & Não informado & Não informado & Não informado & Não informado & Não informado \\
\hline Campos et al. 2013 & Sim & Não informado & Não informado & Não informado & Não informado & Não informado \\
\hline Fisher et al. 2013 & Sim & Não informado & Não informado & Não informado & Sim & Não informado \\
\hline
\end{tabular}

\section{DISCUSSÃO}

Dos sete estudos avaliados, dois estudos tiveram como objetivos verificar se há efeito de interferência de um treinamento em relação ao outro ${ }^{8,11}$, um estudo teve como objetivo verificar o efeito de interferência e da ordem de execução do treinamento ${ }^{14}$, dois estudos investigaram somente o efeito da ordem ${ }^{9,10}$, e por fim, dois estudos tiveram como objetivo verificar o efeito do volume de treinamento combinado, comparando diferentes número de sessões realizadas ao longo de uma semana ${ }^{18,19}$.

Todos os estudos incluídos avaliaram respostas na força muscular e apresentaram melhoras significativas nestas variáveis com o treinamento combinado. Entretanto, três estudos ${ }^{9-11}$ demonstraram que a realização simultânea de um treinamento aeróbico e de força (nesta ordem de execução) pode apresentar um efeito de interferência nas respostas de força muscular de membros inferiores de homens idosos saudáveis. Nestes estudos o grupo que realizou o treinamento combinado (ordem aeróbico-força) apresentou respostas nos parâmetros relacionados a força muscular de membros inferiores significativamente menores do que aqueles que realizaram um treinamento de força isolado ou um treinamento combinado na ordem inversa. Entretanto, cabe salientar que o treinamento aeróbio dos estudos em questão foi realizado em cicloergômetro e em intensidades próximas ao ponto correspondente ao segundo limiar ventilatório, o que pode ter gerado uma fadiga nos músculos dos membros inferiores limitando a sua capacidade de gerar força no treinamento de força subsequente ${ }^{9-11}$. Em contrapartida, um estudo demonstrou que a realização do treinamento combinado, independente da ordem, não apresentou interferência nos ganhos de força de membros inferiores em mulheres idosas saudáveis ${ }^{14}$. Contudo, neste estudo o treinamento aeróbico poderia ser realizado em esteira ou em cicloergômetro, sendo que os autores relatam que a maioria dos sujeitos do estudo optaram pela esteira, e em intensidades entre 60 e $85 \%$ da frequência cardíaca máxima. Avaliando uma população do mesmo sexo e utilizando metodologia de treinamento aeróbio semelhantes, porém treino somente em esteira, Fisher et al. ${ }^{19}$ também não observou o efeito de interferência nos ganhos de força quando o treinamento aeróbio realizado antes.

Neste sentindo, já está descrito na literatura que os exercícios em cicloergômetro apresentam um aumento mais significativo das concentrações de lactato do que a corrida em uma mesma velocidade relativa ao consumo má- 
ximo de oxigênio ${ }^{20}$. Além disso, a modalidade em cicloergômetro apresenta uma maior ativação da rota glicolítica do que a corrida, provavelmente devido a menor massa muscular envolvida para produzir um dado consumo de oxigênio ${ }^{20}$. Os exercícios em bicicleta também apresentam uma maior exigência dos músculos flexores e extensores de joelho e de quadril comparado a corrida. Desta forma uma maior exigência tanto metabólica quanto mecânica pode justificar uma maior interferência nos aumentos da força de membros inferiores

O desempenho aeróbio foi avaliado em seis estudos. Cinco avaliaram através do consumo de oxigênio de pico ou máximo ${ }^{8,9,11,18,19}$ e um estudo avaliou o tempo no teste de 6 minutos $^{14}$. Todos os estudos demonstraram melhoras nestes parâmetros e estas melhoras foram independentes da ordem de execução do treinamento combinado e não houve efeito de interferência do treinamento de força.

Além disso, dois estudos investigaram o volume semanal no treinamento combinado para idosos saudáveis e ambos observaram que volumes de treinos menores apresentam respostas na potência aeróbia e força semelhantes aos volumes maiores ${ }^{18,19}$. No estudo de Ferrari et al. ${ }^{18}$ esse comportamento foi observado em sessões de treinamento combinado em que o treinamento de força e aeróbico eram realizados em uma mesma sessão, enquanto que o estudo de Fisher et al. ${ }^{19}$ os treinamentos eram realizados em dias diferentes, demonstrando que as melhoras parecem ser independentes se a realização do treinamento combinado é feita em um mesmo dia ou em dias distintos.

Concluindo, parece que a melhor forma de prescrição de treinamento combinado para idosos seria a execução do treino de força antecedendo ao treino aeróbio para que não ocorram prejuízos na força muscular de membros inferiores. Entretanto, esse comportamento parece estar relacionado ao tipo de modalidade aeróbia treinada (ex. cicloergômetro e esteira) e também a intensidade do treinamento, sendo esta uma questão de pesquisa que ainda precisa ser melhor investigada. Além disso, pensando em uma melhor aderência ao programa de treinamento pelo idoso, uma frequência semanal de duas vezes de um treinamento combinado apresenta benefícios ao idoso em amplitudes semelhantes a uma frequência maior, e esta pode ser realizada em uma mesma sessão ou em dias diferentes. Por fim, a partir da avaliação dos riscos de viés, cabe salientar que mesmo com os resultados positivos observados na presente revisão sistemática ainda são necessários mais estudos com maior robustez metodológica a fim de obter resultados mais consistentes da eficiência do treinamento combinado para idosos.

\section{REFERÊNCIAS}

1. Deslandes, A. The biological clock keeps ticking, but exercise may turn it back. Arq Neuropsiquiatr. 2013; 71(2): 113-8.

2. Kanegusuku H, Queiroz AC, Chehuen MR, Costa LA, Wallerstein LF, Mello MT, et al. Strength and power training did not modify cardiovascular responses to aerobic exercise in elderly subjects. Braz J Med Biol Res. 2011; 44(9): 864-70.

3. Locks RR, Costa TC, Koppe S, Yamaguti AM, Garcia MC, Gomes AR. Effects of strength and flexibility training on functional performance of healthy older people. Rev Bras Fisioter. 2012; 16(3): 184-90.

4. Matsudo SM, Matsudo VKR, Neto TLB. Impacto do envelhecimento nas variáveis antropométricas, neuromotoras e metabólicas da aptidão física. Rev. Bras. Ciên. e Mov. 8 (4): 21-32, 2000.

5. Filho ETC. Fisiologia do envelhecimento. In: Tratado de Gerontologia, $2^{\mathrm{a}}$ ed., Ed. Atheneu,2007. 
6. Leite LEA, Resende TL, Nogueira GM, Cruz IBM, Schneider RH, Gottlieb MGV. Envelhecimento, estresse oxidativo e sarcopenia: uma abordagem sistêmica. Rev. bras. geriatr. gerontol., v.15, n.2, p.365-380, 2012.

7. Fleck SJ, Kraemer WJ. Fundamentos do treinamento de força muscular. Porto Alegre: Artmed, 2006.

8. Cadore EL, Pinto RS, Pinto SS, Alberton CL, Correa CS, Tartaruga MP, et al. Effects of strength, endurance and concurrent training on aerobic power and dynamic neuromuscular economy in elderly men. J Strength Cond Res. 2011; 25(3): 758-66.

9. Cadore EL, Izquierdo M, Alberton CL, Pinto RS, Conceição M, Cunha G, et al. Strength prior to endurance intra-session exercise sequence optimizes neuromuscular and cardiovascular gains in elderly men. Exp Gerontol. 2012; 47(2): 164-9. doi: 10.1016/j.exger.2011.11.013.

10. Cadore EL, Izquierdo M, Pinto SS, Alberton CL, Pinto RS, Baroni BM, et al. Neuromuscular adaptations to concurrent training in the elderly: effects of intrasession exercise sequence. AGE; 35(3): 891-903, 2012.

11. Cadore EL, Pinto RS, Lhullier FL, Correa CS, Alberton CL, Pinto SS, et al. Physiological effects of concurrent training in elderly men. Int J Sports Med. 2010; 31(10): 689-97.

12. Souza EO, Tricoli V, Roschel H, Brum PC, Bacurau AV, Ferreira JC, et al. Molecular adaptations to concurrent training. Int J Sports Med. 2013; 34(3): 207-13. doi: 10.1055/ s-0032-1312627.

13. Costa LS, Pereira WP, Calixto AM, Abdalla AS, Rosa G. Efeito do exercício aeróbico sobre o desempenho da força de membros inferiores. Braz J Sports Exerc Res. 2010; 1(2): 118-21.

14. Campos ALP, Ponte LSD, Cavalli AS, Afonso MR, Shild JF, Reichert FF, et al. Effects of concurrent training on health aspects of elderly women. Rev Bras Cineantropom Desempenho Hum. 2013: 15(3): 437-47.

15. Mikkola J, Izquierdo M, Gorostiaga EM, Häkkinen K. Neuromuscular and cardiovascular adaptations during concurrent strength and endurance training in untrained men. Int J Sports Med. 2012: 33(9): 702-10.

16. De Morton NA. The PEDro scale is a valid measure of the methodological quality of clinical trials: a demographic study. Aust J Physiother. 2009: 55(2): 129-33.

17. Verhagen AP, de Vet HC, de Bie RA, Kessels AG, Boers M, Bouter LM, Knipschild PG. The Delphi list: a criteria list for quality assessment of randomized clinical trials for conducting systematic reviews developed by Delphi consensus. J Clin Epidemiol. 1998; 51(12): 1235-41

18. Ferrari R, Kruel LFM, Cadore EL, Alberton CL, Izquierdo M, Conceição M, et al. Efficiency of twice weekly concurrent training in trained elderly men. Exp Gerontol. 2013; 48(11): 1236-42.

19. Fisher G, McCarthy JP, Zuckerman PA, Bryan DR, Bickel CS, Hunter GR. Frequency of combined resistance and aerobic training in older women. J Strength Cond Res. 2013; 27(7): 1868-76.

20. Knecthle B, Müller G, Willmann, F, Kotteck K, Eser P, Knecth H. Fat oxidation in men and women endurance athletes in running and cycling. Int J Sports Med. 2004; 25: 38-44.

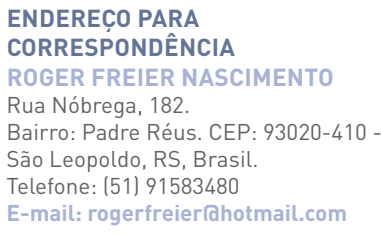

